\title{
Orientated Bacterial Cellulose Culture Controlled by Liquid Substrate of Silicone Oil with Different Viscosity and Thickness
}

\author{
By Ananda PUTRA ${ }^{\dagger}$, Akira KAKUGo, Hidemitsu FURUKAWA, and Jian Ping GoNG*
}

We report the effect of oxygen and viscoelasticity of culture interface on the orientation of bacterial cellulose (BC) fibril synthesized by Acetobacter xylimum (ATCC 53582) at a silicone oil/culture medium (liquid/liquid system) interface. In this work, we used the silicone oil with various kinematic viscosities, $v$ ranging from 10 to 3000 centistokes (CS). We found that the birefringence, $\Delta n$ as a measure of degree of orientation, fibril width, $w$, swelling degree, $q$, and tensile modulus, $E$, increased as increase the thickness and viscosity of silicone oil. However, fracture stress, $\sigma_{\mathrm{F}}$ and fracture strain, $\lambda_{\mathrm{F}}$ decreased with increase the silicone oil thickness and viscosity. Further, we also found that viscoelasticity of culture interface plays an important role to determine the orientation alignment of $\mathrm{BC}$ fibrils. Meanwhile, oxygen did not show significant influence on orientation of $\mathrm{BC}$ fibrils.

KEY WORDS: Bacterial Cellulose / Orientation / Silicone Oil / Oxygen / Thickness / Viscosity /

Bacterial cellulose $(\mathrm{BC})$ is a kind of cellulose produced by bacteria such as Acetobacter xylinum (A. xylinum). BC has excellent mechanical performance including high tensile strength and modulus, high water holding capacity, high moldability, high crystallinity and also high biocompatibility. ${ }^{1,2}$ Currently, BC has received much attention in medical, pharmaceutical and prosthetic applications. ${ }^{3-9}$

As high water holding capacity material, BC gel contains $90 \mathrm{wt} \%$ or more of water. However, the water is easily squeezed out from the gel after low compression such as only by finger and no more recovery in the swelling property due to hydrogen-bond formation between cellulose fibrils. We have developed biocompatible hydrogels with a high reversible swelling ability after repeated compression by combining BC with a natural hydrophilic polymer such as gelatin using the semi-inter penetrating network (semi-IPN) technique called Double Network (DN). ${ }^{10}$ The fracture strength and the elastic modulus of the BC-gelatin gel under compressive stress were in the order of megapascals that are almost equivalent to those of articular cartilage. ${ }^{11}$

BC gel synthesized by the aerobic bacterium of $A$. xylinum and cultivated under static immersion has a randomly oriented fibril network structure in the form of a gel at the air-liquid interface of the culture medium. With regard to the structure of $\mathrm{BC}$ gel, the production of $\mathrm{BC}$ gel having a well-oriented fibril arrangement could be beneficial for its use as, for example, a substrate for cell culture or substitute material for soft tissue replacement.

Previously, we reported a novel one-pot method for the production of $\mathrm{BC}$ gel having highly oriented fibril structure by cultivation of the $\mathrm{BC}$ on oxygen-permeable polydimethylsiloxane (PDMS) substrate with ridges of various sizes. ${ }^{12}$ Uni- axially oriented fibrils of $\mathrm{BC}$ gel was obtained on the ridges PDMS, where the degree of orientation of fibrils increased with a decrease in the ridge wavelength of PDMS substrate and reached maximum at ridge wavelength of $4.5 \mu \mathrm{m}$, a size that is believed to be determined by the size of bacteria. This BC gel showed higher birefringence $(\Delta n)$, fracture stress $(\sigma)$, and swelling degree $(q)$, broader fibrils size, and lower elastic modulus $(E)$ than the BC cultured on air-liquid interface. Interestingly, localized orientation of fibrils was even observed on un-ridged smooth PDMS surface. Based on this technique, we also successfully synthesized a tubular BC with oriented fibrils alignment. ${ }^{13}$ This technique might be useful to enhance the potential application of the BC. However, the mechanism of orientation induced by A. xylinum during secretion of cellulose fibril was not understood yet. We believed that there must be several factors which might induce the orientation of cellulose fibrils by A. xylinum such as the component of cultivation (medium composition), $\mathrm{pH}$, oxygen, temperature, type of culture method/cultivation design used, and behavior of culture interface. It is imperative to identify and characterize the dominant factors that induce the orientation of the BC fibril.

In this paper, we study the dominant factors to induce the orientation of fibrils in the BC in terms of oxygen availability and viscoelasticity of the culture substrate. In order to study these factors, we used silicone oil cover with different kinematic viscosity, $v$, as a liquid substrate, on which the $\mathrm{BC}$ was cultured. Figure 1 shows the interesting effect of the viscosity of the silicone oil substrate. As shown in the Figure $1 \mathrm{~b}$, the bacteria produce the $\mathrm{BC}$ culture on the silicone oil substrate, where the upside of culture medium was covered with the silicone oil substrate. Interestingly, the orientation of the $\mathrm{BC}$ fibrils was increased as the viscosity of the silicone oil

\footnotetext{
Department of Biological Sciences, Graduate School of Science, Hokkaido University, Sapporo 060-0810, Japan

Present address: Advanced Science Research Center, Japan Atomic Energy Agency, Tokai 319-1195, Japan

*To whom correspondence should be addressed (Tel: +81-11-706-2774, Fax: +81-11-706-2774, E-mail: gong@sci.hokudai.ac.jp).
} 


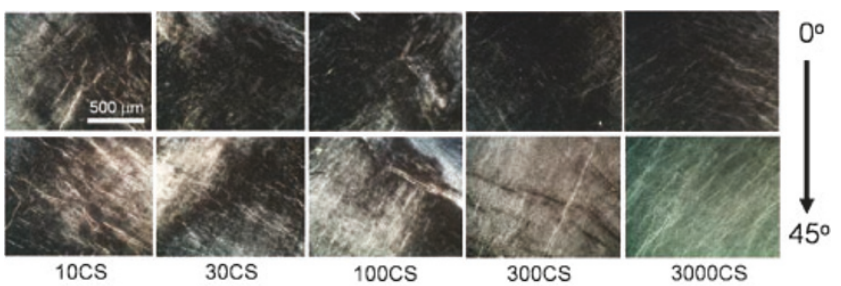

(a)

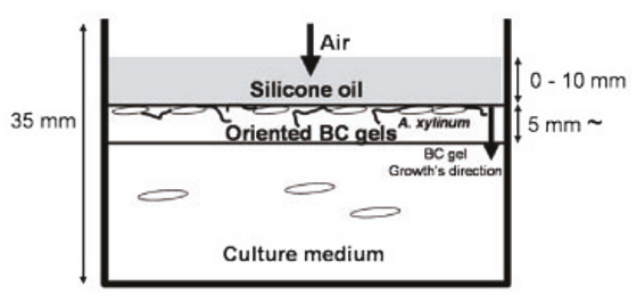

Matrix reservoir cultivation

(b)

Figure 1. Optical polarizing microscope images of $\mathrm{BC}$ gel cultured at various kinematic viscosities of silicone oil, KF-96 (10, 30, 100, 300, 3000)CS with the oil thickness of $2 \mathrm{~mm}$ (a) and, the schematic illustration of matrix reservoir cultivation for oriented BC culture controlled by liquid substrate of silicone oil with different viscosity and thickness (b).

increases (Figure 1a). In order to clarify the effect of the silicone oil substrate, the structure of BC-gel culture was characterized by Scanning Electron Microscope (SEM) and Optical Polarizing Microscope (OPM). Also, the mechanical properties of the $\mathrm{BC}$ gels were characterized by tensile strength measurement. Then, we will demonstrate that oxygen availability has no significant effect, however viscosity plays an important role in the orientation alignment of the BC fibrils in the $\mathrm{BC}$ gels. This result implies that the substrate-controlled mobility of the A. xylinum during secretion of $\mathrm{BC}$ fibrils strongly affects on the properties of the $\mathrm{BC}$ culture due to the orientation of the $\mathrm{BC}$ fibrils.

\section{EXPERIMENTAL}

\section{Materials}

Silicone oil with various kinematic viscosities, $v$ of 10,30 , 100, 300, and 3000 centistokes, CS (KF96-10, 30, 100, 300, 3000CS, (Shin Etsu)) was use as purchased. Bacteria of Acetobacter xylinum, American Type Culture Collection (ATCC) 53582 were used. Culture medium was used based on Hestrin $\mathrm{S}$ and Schramm medium (wt.-\%); ${ }^{14} \mathrm{~g}$ bacto peptone (difco bacto peptone, Becton dickinson and Co, USA), $5 \mathrm{~g}$ yeast extracts (Difco Becton dickinson and Co, Ltd), $2.7 \mathrm{~g}$ disodium hydrogen phosphate $\mathrm{Na}_{2} \mathrm{HPO}_{4}$ (Junsei Chemical Co. Ltd), $1.15 \mathrm{~g}$ citric acid (Wako pure chemical Ltd), and $20 \mathrm{~g} \mathrm{D} \mathrm{(+)} \mathrm{glucose} \mathrm{(Wako} \mathrm{pure} \mathrm{chemical}$ Industries, Ltd), and $\mathrm{HCl}$. Aqueous solution of $\mathrm{NaOH}$ was used for purification. Indigocarmine, glycerin, glucose, $\mathrm{KOH}$, Ar gas were used for study the diffusivity of oxygen through silicone oil.

\section{Production of BC Gel}

Sterile-Hestrin-Schramm medium (pH 6) and a cell suspension prepared from A. xylinum culture (ATCC 53582) with volume ratio of $10: 1$ were poured into sterile-vessel $(\phi=$ $8.5 \mathrm{~cm})$. For study the effect of oxygen, the amount of autoclaved silicone oil, KF96-100CS was poured gently on the surface of medium up to various thicknesses of 1, 2, 4, 6, 8 and $10 \mathrm{~mm}$. For study the effect of viscoelasticity of the culture interface, the amount of autoclaved silicone oil with various kinematic viscosities (10, 30, 100, 300, and 3000CS) were poured gently on the surface of medium up to $2 \mathrm{~mm}$ in thickness. These samples were incubated at temperature of $28^{\circ} \mathrm{C}$ in static condition for $7 \mathrm{~d}$. The schematic illustration of matrix reservoir cultivation of this $\mathrm{BC}$ is shown in Figure $1 \mathrm{~b}$. The obtained gel-like BC were purified by soaking into a large amount of distilled water for one day (the water was changed 3 times), and then autoclave in $1 \%(\mathrm{w} / \mathrm{v})$ aqueous solution of $\mathrm{NaOH}$ at $121{ }^{\circ} \mathrm{C}$ for 20 min to remove bacterial cell debris and alkali-soluble components. After reached room temperature, the $\mathrm{BC}$ gel were then washed with distilled water several times and soaked into distilled water for a prolonged time to $\mathrm{pH} 7$ and stored in distilled water at room temperature prior to use. As comparison, BC gel was also prepared on a $1 \mathrm{~mm}$ thickness of flat and smooth surface of PDMS sheet. BC gel cultured on air (without silicone oil) was also prepared by similar process.

\section{Measurement}

The amount of water contained in the BC gels was characterized by the degree of swelling, $q$, which is defined as the weight ratio of as-prepared swollen sample to dry sample. Dry BC gels were obtained by drying the sample in vacuum at $60^{\circ} \mathrm{C}$ until a constant weight was obtained.

Optical polarizing microscope observation was performed using an Olympus BH-2. Samples of BC gels were placed on pre-cleaned micro slide glass plate $(76 \times 26 \times(0.8-1.0) \mathrm{mm})$ (Matsunami), and upper free surfaces were observed. The birefringence, $\Delta n=n_{\text {parallel }}-n_{\text {perpendicular }}$, of the $\mathrm{BC}$ gel was measured by an optical polarizing microscope with Berek compensator. ${ }^{15}$ The sample orientation direction was determined by turning the sample under the optical polarizing microscope. Under these conditions, $\Delta n$ was measured from retardation. The images were taken at $0,45,90,135$ and 180 degrees. Average $\Delta n$ was determined by measuring $\Delta n$ several times for each sample. During observation, the samples were in wet conditions.

Morphology and microstructure of BC gel were examined by Scanning Electron Microscope, SEM (HITACHI-S-2250N). Prior to observe with SEM, small pieces of BC gels were freeze dried (VirTis Advantage AD2.0 XL-SC) for one day and mounted on SEM stub using double tip, and then sputter-coated with palladium (60 to $90 \mathrm{~s}$ ) by ions sputtering (HITACHI-E 1010) to avoid electrical charging. The images was taken at $15 \mathrm{kV}$ accelerate voltage.

The tensile strength tester (TENSILON, Orientec Co.) was used to measure the mechanical properties of $\mathrm{BC}$ gel. The wet samples of BC were cut into dumbbell shapes (dowel width, 
thickness and length of $2,0.9,12 \mathrm{~mm}$, respectively) and stretched using a clamp attachment at a strain rate of $10 \% \mathrm{~min}^{-1}$ on the open air. The strain rate was referenced to the initial length of the specimens. Failure points of the tensile strength were determined from the peak of the stress-strain curve. The tensile modulus, $E$, was determined by the average slope over the strain ratio range of $0-10 \%$ from the stress-strain curve.

\section{Diffusivity of Oxygen Passes through Silicone Oil}

The diffusivity of oxygen through silicone oil was studied based on a color change of water containing oxygen indicator solution below the silicone layer induced by an oxidationreduction process of indigocarmine. In this oxidation-reduction process of indigocarmine, oxygen acted as oxidizing agent and glucose acted as reducing agent. Color changes of indigo carmine depend on the availability of oxygen in the solution. In the oxidation state, indigocarmine turn to several different colors from red (low oxygen) to purple, blue and green (high oxygen). Meanwhile, in the reduction state, it turns to blue, purple, red and yellow. The yellow color indicates no oxygen left in the solution. The following is a simple mechanism for the oxidation and reduction process of the indigocarmine. ${ }^{16}$

$$
\begin{gathered}
\mathrm{CH}+\mathrm{OH}^{-} \rightleftarrows \mathrm{C}^{-}+\mathrm{H}_{2} \mathrm{O} \\
\mathrm{O}_{2}+\mathrm{D} \stackrel{\text { fast }}{\longrightarrow} \mathrm{D}_{\mathrm{ox}} \\
\mathrm{D}_{\mathrm{ox}}+\mathrm{C}^{-} \stackrel{\text { slow }}{\longrightarrow} \mathrm{X}^{-}+\mathrm{D}
\end{gathered}
$$

where $\mathrm{CH}$ is of glucose, $\mathrm{D}$ is reduced indigocarmine, $\mathrm{D}_{\mathrm{ox}}$ is oxidized indigocarmine, and $\mathrm{X}^{-}$represent the oxidation products from glucose. So, once there is oxygen in solution, immediately indigocarmine become in the oxidized stated $\left(D_{o x}\right)$ and the color of the solution change to red, purple or blue depend on amount of oxygen in the solution. For a long time, all the $\mathrm{D}_{\mathrm{ox}}$ is slowly reduced by glucose, and the color of solution will turn to yellow. From this color changing, relative amount of diffusive oxygen (color pixel of solution's color) and relative diffusion flux (pixel/second) of oxygen can be calculated.

Prior to study the diffusivity of oxygen through silicone oil, water, silicone oil and oxygen indicator solution were degassed in the glove box and then were left under Ar gas atmosphere for at least $2 \mathrm{~d}$ to minimize the oxygen level in the solution. Oxygen indicator solution was prepared as follows: Into $150 \mathrm{~mL}$ of glycerin, $0.8 \mathrm{~g}$ of glucose and $0.12 \mathrm{~g}$ indigocarmine which was firstly diluted in $10 \mathrm{~mL}$ of water was added. This solution was called solution I. Another solution of $\mathrm{KOH}$ was prepared by dissolved $37.5 \mathrm{~g}$ of $\mathrm{KOH}$ in $100 \mathrm{~mL}$ of water, called solution II. Mixture of solution I and II was called oxygen indicator solution.

Under Ar gas atmosphere in the glove box, solution I and II was mixed with a ratio of $4: 1$, then $2 \mathrm{~mL}$ of this oxygen indicator solution was added to $10 \mathrm{~mL}$ of distilled water in a $50 \mathrm{~mL}$ bottle and mixed gently until homogeneous colored solution appeared. It is noted that if red color appears it indicates that in the water still remains small amount of oxygen which can oxidize the indigocarmine in the oxygen indicator. On to this solution, silicone oil, KF96-100CS was added with various amount to reach a thickness of $0,1,2,4,6,8$ and $10 \mathrm{~mm}$. Silicone oil of various viscosity $(10,30,100,300$, and 3000CS) was also added with amount to reach a thickness of $2 \mathrm{~mm}$. If no more oxygen left in the solution, the indicator started to be reduced by the glucose which acted as reducing agent, and the solution turned to yellow color. Then the mixtures of these solution were take out from the glove box and were left unshaken several minutes to allow oxidizing process of indicator by remaining oxygen in the solution. After the yellow color appeared completely, the solution was shaken gently by using magnetic stirrer to diffuse oxygen pass through the oil phase in to the water phase. Diffusion of oxygen was indicated by changing the color of solution from yellow to red due to the oxidation of indigo carmine by oxygen. The amount of diffusive-oxygen was recorded as relative diffusion in color pixels as a function of time and was calculated based on the changing of color density using computer program of windows image-pro plus 6.1.

\section{RESULTS AND DISCUSSION}

\section{Effect of Oxygen: Varying in Silicone Oil Thickness}

The BC gel cultured on silicone oil/culture medium (BC liquid/liquid) interface was very transparent. Either at high or low thickness of silicone oil, the transparence was higher than that of BC cultured at air/liquid (BC-air) interface or at PDMS solid/liquid interface (BC solid/liquid) (Figure 2a). Production of BC gel on liquid/liquid was at low amount of oxygen due to low permeability of oxygen passed through the liquid compare to that directly on air/liquid in atmospheric conditions. By increasing the thickness of the oil, the diffusivity of $\mathrm{O}_{2}$ became slower. We found that the thickness of BC decreased as increase the silicone oil thickness for a certain culture time (data not shown). This result indicated that the oxygen affects the productivity of the $\mathrm{BC}$.

BC gel cultured on various thickness of the silicone oil showed an isotropic and strong birefringence which can be seen as colorful images when was viewed under optical polarizing microscope (Figure 2b, 2c, column II). The birefringence $(\Delta n)$, as an indicator for the degree of orientation increased linearly as increase the silicone oil thickness (Figure 3a). The $\Delta n$ of these $\mathrm{BC}$ was more than 10 times higher than that of BC-air/liquid. SEM image also clearly indicated that the orientation of $\mathrm{BC}$ fibrils in $\mathrm{BC}$-liquid/liquid is higher than that of BC-air/liquid. Even though the persistence length of the fibril could not be measured, the average fibril width slightly increased $(94 \mu \mathrm{m}$ to $105 \mu \mathrm{m})$ (Figure 2d, column II and 3a). Further, the swelling degrees of the $\mathrm{BC}$ also increased with increase the silicone oil thickness (Figure 3b).

We also determined the tensile strength properties of these $\mathrm{BCs}$ (Figure 3b, c). The strength of those BC gels was different from each other. Tensile modulus increased as the silicone oil thickness increased (0.06 to $0.95 \mathrm{MPa})$, meanwhile there was no significant changing in the tensile fracture stress, but the fracture strain linearly decreased as the silicone oil thickness 
BC- air/liquid

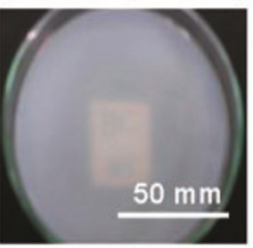

BC- liquid/liquid

II

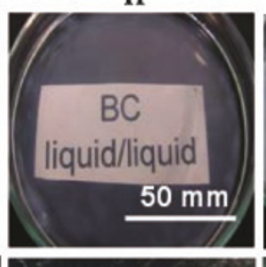

(b)
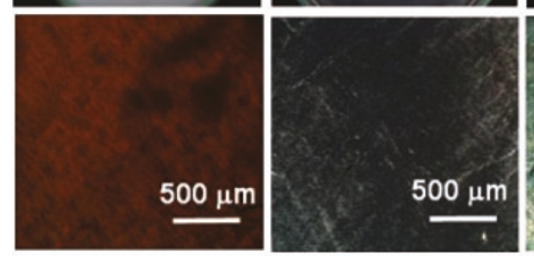

(c)

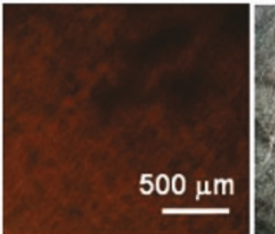

(d)
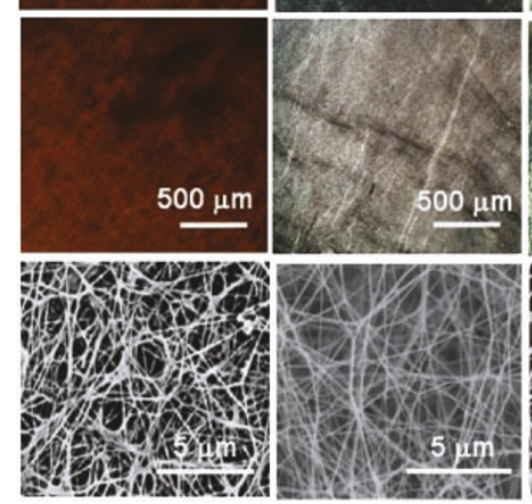

Figure 2. The bright field images (a), optical polarizing microscope images at 0 degree (parallel to the substrate ridged direction) (b), and 45 degree (c), and SEM images (d) of BC gels produced at air/liquid (column I), at liquid/liquid (column II) with silicone oil KF-96100CS thickness of $2 \mathrm{~mm}$, and at solid/liquid (column III) with PDMS thickness of $1 \mathrm{~mm}$.

increased $(0.46$ to $0.28 \mathrm{~mm} / \mathrm{mm})$. This tensile strength tendency might be determined by the water content (swelling degree), fibril width and the orientation of fibril of BC gels. Generally, tensile modulus of a material depends on its water content, where, tensile modulus will decrease as increase its swelling degree. In our result, swelling degrees of BC gel cultured at silicone oil with various thicknesses were almost similar each other (Figure 3b). Therefore, increasing the tensile modulus might be not significantly determined by the swelling degree (water content), but it might be determined by the fibril width and its orientation. The fibril width and the $\Delta n$ of the BC increased as increase the thickness of silicone oil (Figure 3a).

\section{Effect of Interface: Varying in Kinematic Viscosity, $v$ of Silicone Oil}

In our previous work, we have shown that well oriented fibrils of BC were obtained at ridge solid/liquid interface. ${ }^{12}$ At smooth solid/liquid interface, fibrils with local orientation were also obtained (Figure 2, column III). It is not clear yet why at smooth interface, BC showed such orientation. To elucidate this, we investigated the kinematic viscosity effect of silicone oil on the fibril orientation of the BC. Even though the silicone oil was softer and movable than solid, its surface was smoother and more homogenous than solid.

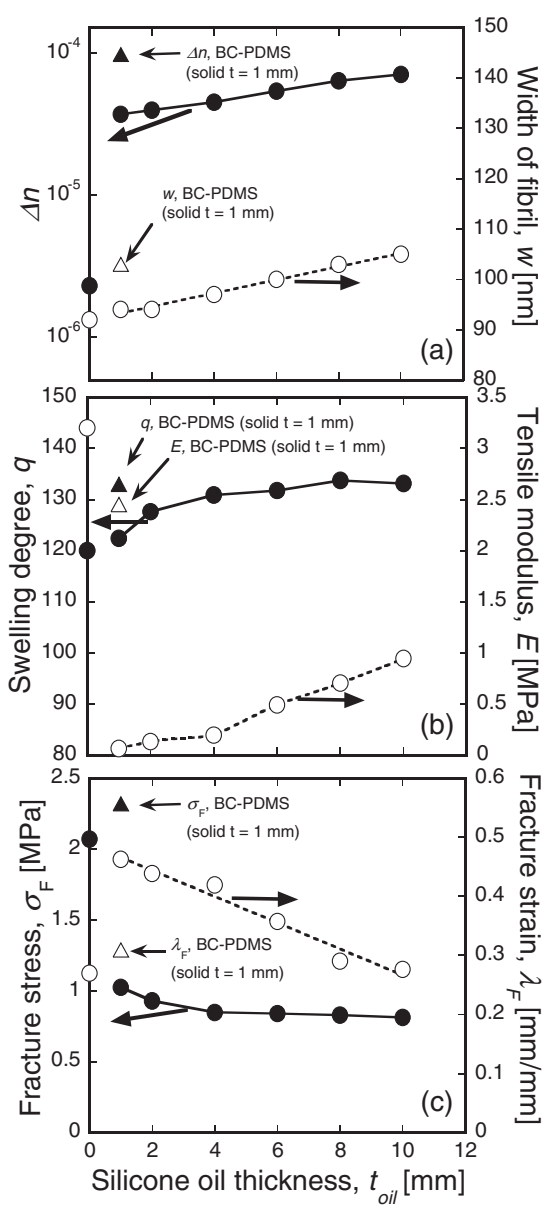

Figure 3. Dependence of birefringence, $\Delta n(O)$ and width of fibrils, $w(\bigcirc)$ (a), swelling degree, $q(\mathbf{O})$ and tensile modulus, $E(O)(\mathrm{b})$, and fracture stress $\sigma_{F}(\Omega)$ and fracture strain, $\lambda_{F}(O)$ (c) of BC gels obtained between silicone oil, KF-96-100CS $(v=100 \mathrm{CS})$ of various thicknesses and culture liquid interface. The curves were shown to guide the eyes.

The BC gel cultured on silicone oil of various kinematic viscosities was very transparent, similar to that of on silicone oil of various thicknesses. Either at high or low kinematic viscosities of silicone oil, the transparency was higher than that of BC-air or BC solid/liquid. We also found that the thickness of $\mathrm{BC}$ decreased as increase the kinematic viscosity of silicone oil. These results were similar to the result of varied in silicone oil thickness, except that the values were different.

BC gel cultured on silicone oil of various kinematic viscosities also showed an isotropic and strong birefringence which could be observed by colorful images when they were viewed under optical polarizing microscope (Figure 1a). The birefringence $(\Delta n)$ increased as increase the kinematic viscosity of silicone oil (Figure 4a). These results showed that the interface really affected the fibrils orientation of BC. Mean while, even though the persistence length of the fibril could not be measured, we found that no significant change in the average fibril width at low kinematic viscosity (lower than 100CS), but it drastically increased as kinematic viscosity of silicone oil increased $(93 \mu \mathrm{m}$ to $110 \mu \mathrm{m})$ (Figure $4 \mathrm{a}$ ). The 

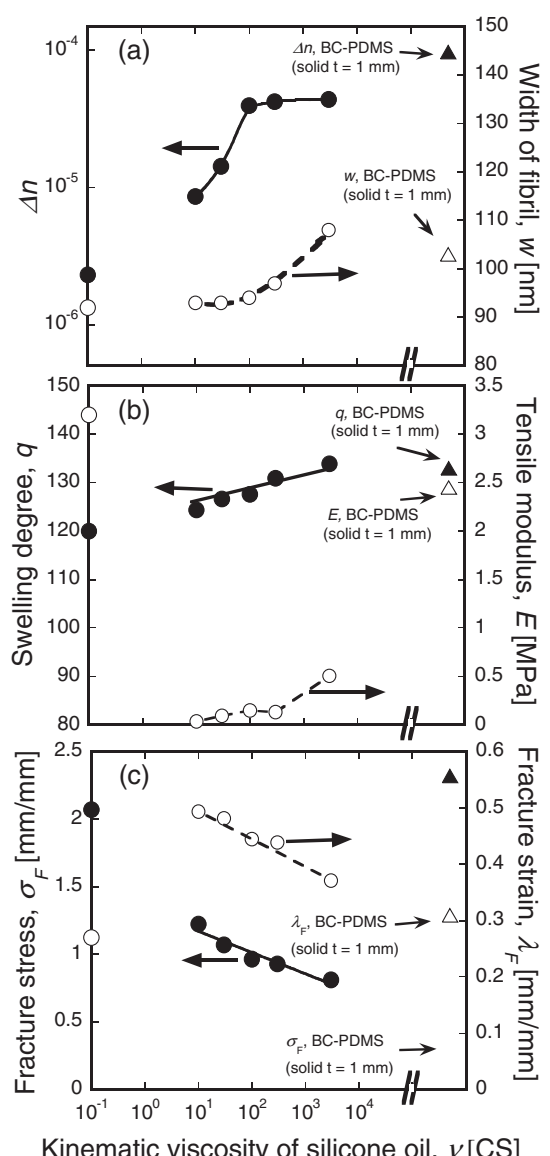

Figure 4. Dependence of birefringence, $\Delta n(O)$ and width of fibrils, $w(O)$ (a), swelling degree, $q(O)$ and tensile modulus, $E(O)(b)$, and fracture stress $\sigma_{F}(\mathbf{O})$ and fracture strain, $\lambda_{F}(\bigcirc)(\mathrm{c})$ of $\mathrm{BC}$ gels obtained between silicone oil of various kinematic viscosities at constant thickness $(t=2 \mathrm{~mm})$ and culture liquid interface. The curves were shown to guide the eyes.

swelling degrees of the $\mathrm{BC}$ increased with increase in the kinematic viscosity of silicone oil (Figure $4 \mathrm{~b}$ ). Tensile modulus increased with increase in the kinematic viscosity of silicone oil thickness (0.04 to $0.51 \mathrm{MPa}$ ) (Figure $4 \mathrm{~b}$ ). In contrary, the tensile fracture stress and fracture strain decreased with increased in the viscosity of silicone oil (Figure 4c). This result was almost similar to the BC cultured on silicone oil of various thickness/liquid interfaces.

\section{Diffusivity of Oxygen Pass through Silicone Oil}

In order to clarify the dominant factor (oxygen availability or viscoelasticity of culture interface) to induce the orientation of fibrils in BC gel, we determined the diffusivity of oxygen pass through the silicone oil phase with various thickness and viscosity. Schematic illustration of diffusivity of oxygen pass through silicone oil is shown in Figure 5. As already mentioned in the experimental section, preparation of sample was done in the glove box under Argon atmospheric condition and the diffusion of oxygen pass through the silicone oil phase was done in open air by stirring the solution simultaneously.

Figure 6a shows the time course of the relative diffusion of oxygen passed through silicone oil, KF-96-100CS phase with various thicknesses into water which contains oxygen indicator solution. It is clearly seen that the amount of oxygen diffused through silicone oil phase into water decreased as increase the silicone oil thickness. This diffusion of oxygen was very much lower than that without applying silicone oil on the water surface. Similar result was observed for silicone oil with various kinematic viscosities at a constant thickness $(\mathrm{t}=2$ $\mathrm{mm}$ ) (Figure 7a). The flux of oxygen, estimated from initial slope of curves in Figures 6a and 7b (below $600 \mathrm{~s}$ ) decreased as increase in the silicone oil thickness and kinematic viscosities (Figures $6 \mathrm{~b}$ and $7 \mathrm{~b}$ ). Thus, increasing the silicone oil thickness and kinematic viscosity found to be decreasing the diffusivity of oxygen passed through it.

Figure 8 shows a curve between relative fluxes of oxygen against the $\Delta n$. The figure shows that the $\Delta n$ of $\mathrm{BC}$ gels cultured at the interface of culture medium and silicone oil with various thicknesses 1 to $10 \mathrm{~mm}$ only slightly changed. This indicates that oxygen availability was not the dominant factor for creating an orientation of fibrils. However, the $\Delta n$ of BC gels drastically increased with decreasing relative flux of oxygen by changing the viscosity of the silicone oil from low to high value. We observed that the silicone oil of KF96-100CS was the critical viscosity to obtain oriented $\mathrm{BC}$ gel. At kinematic viscosity of silicone oil higher than that of the critical kinematic viscosity, there was no significant changed in the $\Delta n$. This result indicates that culture interface is a dominant factor to inducing the orientation of BC fibrils. This might be explained as follows; the A. xylinum moved freely at the interface between culture medium and air and produce $\mathrm{BC}$ with random orientation. When the interface was changed to culture medium and liquid (silicone oil), the motion of A. xylimum will be restricted. At low kinematic viscosity of liquid, small restriction in the movement of $A$. xylinum occurred, which led to $\mathrm{BC}$ with random orientation that was similar to $\mathrm{BC}$-air. However, by increasing the kinematic viscosity of the silicone oil to the critical point, strong restriction in the movement occurred, which led to the drastic increase in $\Delta n$ by unknown process. At kinematic viscosity higher than that of the critical viscosity value, there was no significant effect on the $\Delta n$ of BC gel. Thus, finally we can say that the culture interface is the dominant factor to obtain the BC gel with oriented fibrils.

\section{CONCLUSIONS}

The effect of oxygen and culture interface on the orientation of $\mathrm{BC}$ fibril has been investigated by culturing $\mathrm{BC}$ on silicone oil with different thickness and viscosity. The degree of orientation, $\Delta n$, fibril width, $w$, swelling degree, $q$, and tensile modulus, $E$, increased as increase the thickness and viscosity of silicone oil. However, fracture stress, $\sigma_{\mathrm{F}}$ and fracture strain, $\lambda_{\mathrm{F}}$ decreased with increase the silicone oil thickness and viscosity. Further, we found that viscoelasticity of culture interface (silicone oil) plays an important role to determine the orientation alignment of BC fibrils. Meanwhile, oxygen did not show significant influence for creating an oriented $\mathrm{BC}$ fibrils. 
A. Preparation of sample in the Glove box under Ar gas atmospheric conditions

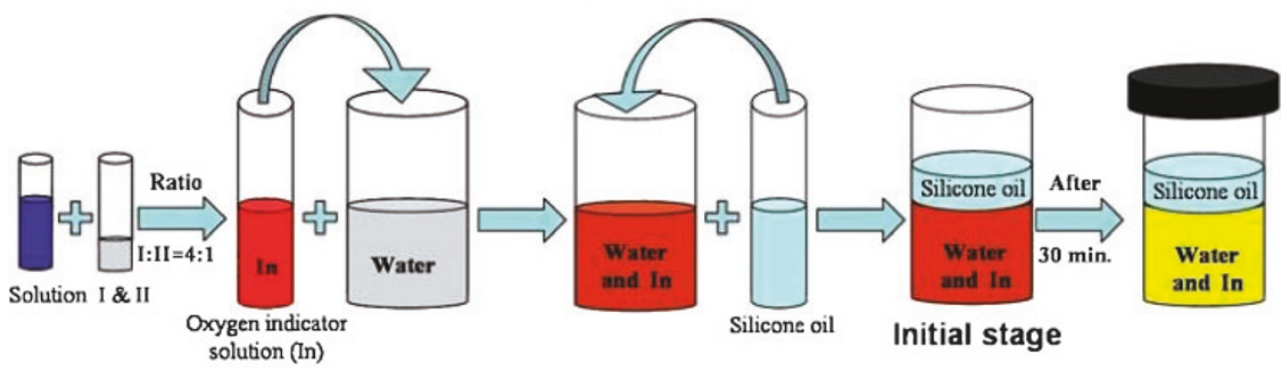

B. Diffusion of Oxygen pass through silicone oil phase into water in open air

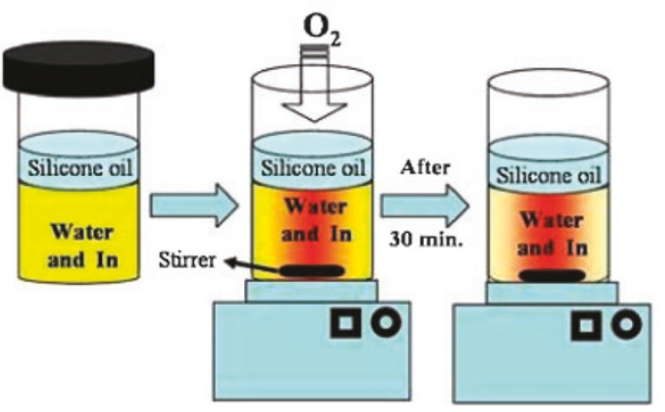

Figure 5. Schematic illustrations of diffusivity of oxygen pass through silicone oil. A) Color changing images of oxygen indicator (indigo carmine) in water with silicone oil on the surface in glove box under Argon atmospheric condition and B) just exposed in atmospheric condition from Argon atmosphere. Yellow color indicates no $\mathrm{O}_{2}$ left in the solution and the red color indicates $\mathrm{O}_{2}$. The color change from yellow to red indicates the diffusion of $\mathrm{O}_{2}$ through silicone oil phase.
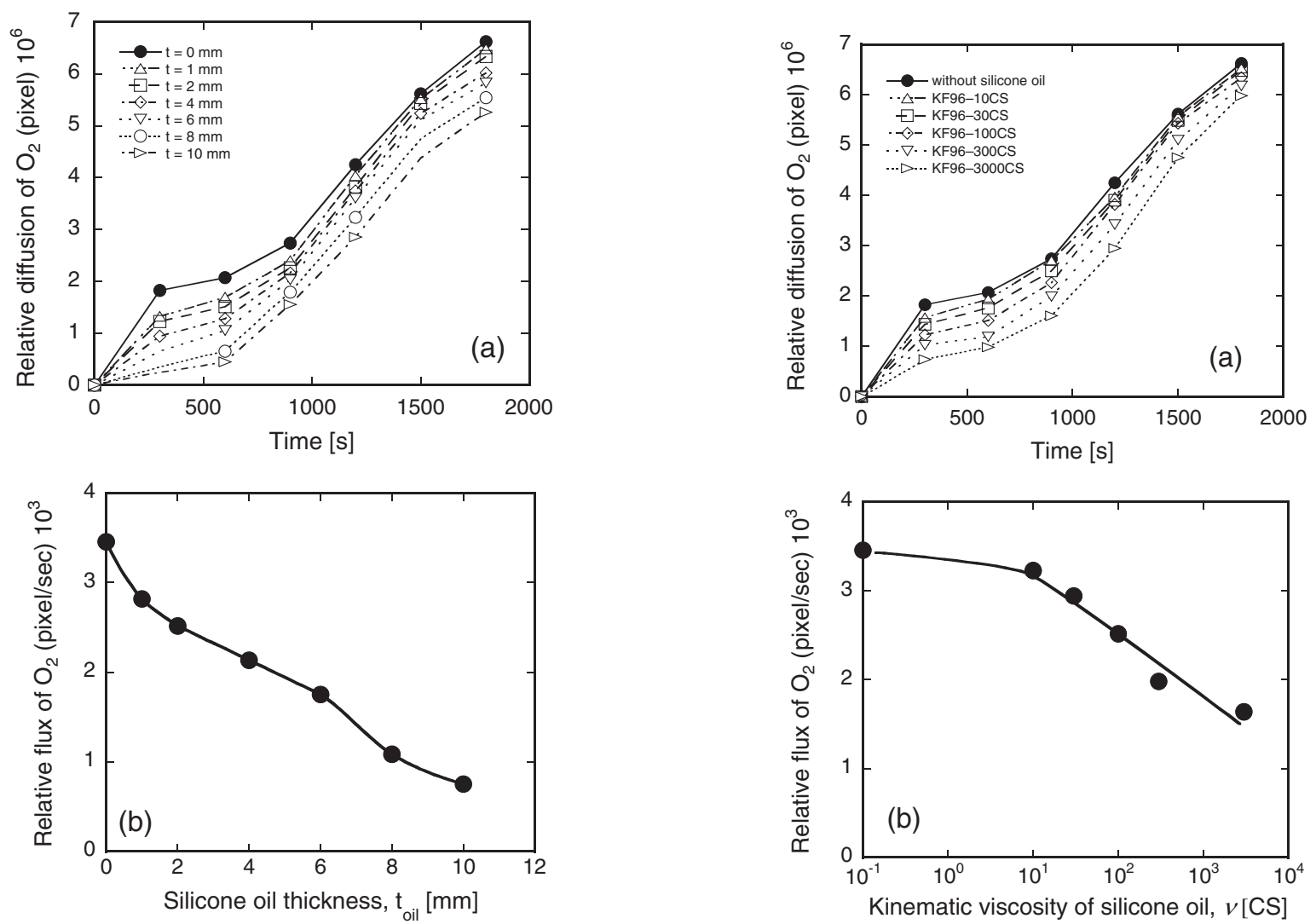

Figure 6. The relative diffusivity of oxygen which passed through the silicone oil, KF-96-100CS ( $v=100 \mathrm{CS}$ ) with various thicknesses; (a) the time course of the relative diffusion and (b) relative flux of oxygen, estimated from the slope of curves of (a).

Figure 7. The relative diffusivity of oxygen which passed through the silicone oil with various kinematic viscosities at constant thickness $(t=$ $2 \mathrm{~mm}$ ); (a) the time course of the relative diffusion and (b) relative flux of oxygen, estimated from the slope of curves of (a). 


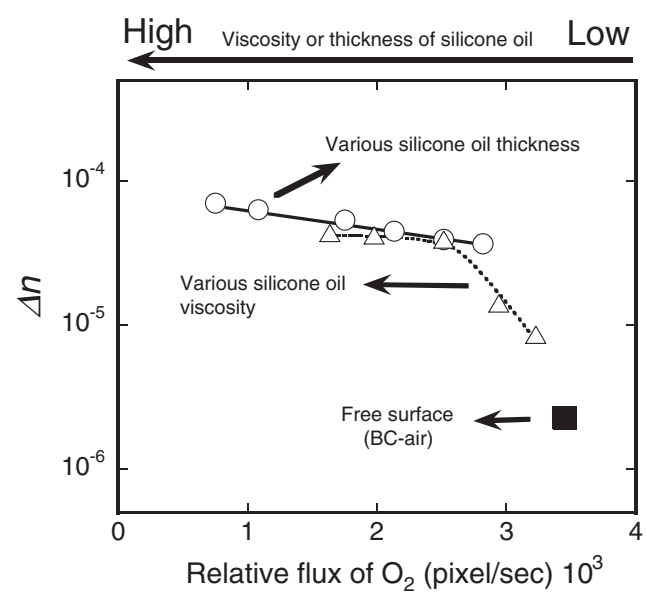

Figure 8. Dependence of $\Delta n$ on the relative flux of oxygen diffused through silicone oil for two batches of experiment, $(O)$ : silicone oil, KF-96$100 \mathrm{CS}(v=100 \mathrm{CS})$ with various thicknesses at constant kinematic viscosity, $(\boldsymbol{\Delta})$ : various kinematic viscosities of silicone oil at a constant thickness $(t=2 \mathrm{~mm})$. ( $\square$ ) is the result for free surface.

Acknowledgment. This research was financially supported by the Ministry of Education, Science, Sports, and Culture, Japan (Grand-in-Aid of Specially Promoted Scientific Research).

Received: January 27, 2009

Accepted: June 1, 2009

Published: July 15, 2009

\section{REFERENCES}

1. D. Klemm, B. Heublein, H. P. Fink, and A. Bohn, Angew. Chem., Int Ed., 44, 3358 (2005).

2. A. Okiyama, M. Motoki, and S. Yamanaka, Food Hydrocolloids, 6 , 493 (1993).

3. W. Czaja, A. Krystynovicz, S. Bielecki, and R. M. Brown, Jr., Biomaterials, 27, 145 (2006).

4. J. D. Fontana, C. G. Joerke, M. Baron, M. Maraschin, A. G. Ferreira, and I. L. Torriani, Appl. Biochem. Biotechnol., 63-65, 327 (1997).

5. J. D. Fontana, A. M. D’Souza, C. K. Fontana, I. L. Torriani, J. C. Moreschi, and B. J. Gallotti, Appl. Biochem. Biotechnol., 24-25, 253 (1990).

6. D. Klemm, D. Schumann, U. Udhart, and S. Marsh, Prog. Polym. Sci., 26, 1561 (2001)

7. A. M. Sokolnicki, R. J. Fisher, T. P. Harrah, and D. L. Kaplan, J. Membr. Sci., 272, 15 (2006).

8. K. Watanabe, Y. Eto, S. Takano, S. Nakamori, H. Shibai, and S. Yamanaka, Cytotechnology, 13, 107 (1993).

9. A. Svensson, E. Nicklasson, T. Harrah, B. Panilaitis, D. S. Kaplan, M. Brittberg, and P. Gatenholm, Biomaterials, 26, 419 (2005).

10. A. Nakayama, A. Kakugo, J. P. Gong, Y. Osada, M. Takai, T. Erata, and S. Kawano, Adv. Funct. Mater., 14, 1124 (2004).

11. C. W. Mc. Cutchen, in "The Joints and Synovial Fluid," L. Sokoloff, Ed., New York, Academic, 1978, Chapter 10 .

12. A. Putra, A. Kakugo, H. Furukawa, J. P. Gong, Y. Osada, T. Uemura, and M. Yamamoto, Polym. J., 40, 137 (2008).

13. A. Putra, A. Kakugo, H. Furukawa, J. P. Gong, and Y. Osada, Polymer, 49, 1885 (2008).

14. S. Hestrin and M. Schramm, Biochem. J., 58, 345 (1954).

15. M. Born and E. Wolf, "Principles of Optics," Cambridge UK, Cambridge University Press, 1959.

16. A. G. Cook, R. M. Tolliver, and J. E. Williams, J. Chem. Educ., 71, 160 (1994). 